\title{
Tunable photonic bandgap fiber based devices for optical networks
}

Alkeskjold, Thomas Tanggaard; Scolari, Lara; Rottwitt, Karsten; Bjarklev, Anders Overgaard; Peucheret, Christophe

Published in:

ICTON 2005 proceedings

Link to article, DOI:

10.1109/ICTON.2005.1505770

Publication date:

2005

Document Version

Publisher's PDF, also known as Version of record

Link back to DTU Orbit

Citation (APA):

Alkeskjold, T. T., Scolari, L., Rottwitt, K., Bjarklev, A. O., \& Peucheret, C. (2005). Tunable photonic bandgap fiber based devices for optical networks. In ICTON 2005 proceedings (pp. 140-143). IEEE.

https://doi.org/10.1109/ICTON.2005.1505770

\section{General rights}

Copyright and moral rights for the publications made accessible in the public portal are retained by the authors and/or other copyright owners and it is a condition of accessing publications that users recognise and abide by the legal requirements associated with these rights.

- Users may download and print one copy of any publication from the public portal for the purpose of private study or research.

- You may not further distribute the material or use it for any profit-making activity or commercial gain

- You may freely distribute the URL identifying the publication in the public portal 


\title{
Tunable Photonic Bandgap Fiber Based Devices for Optical Networks
}

\author{
Thomas T. Alkeskjold, Lara Scolari, Karsten Rottwitt, Anders Bjarklev and Christophe Peucheret \\ Research Center COM, Technical University of Denmark \\ Building 345v,2800 Lyngby, Denmark. Contact: kar@com.dtu.dk
}

\begin{abstract}
In future all optical networks one of the enabling technologies is tunable elements including reconfigurable routers, switches etc. Thus, the development of a technology platform that allows construction of tuning components is critical. Lately, microstructured optical fibers, filled with liquid crystals, have proven to be a candidate for such a platform. Microstructured optical fibers offer unique wave-guiding properties that are strongly related to the design of the air holes in the cladding of the fiber. These wave-guiding properties may be altered by filling the air holes with a material, for example a liquid crystal that changes optical properties when subjected to, for example, an optical or an electrical field. The utilization of these two basic properties allows design of tunable optical devices for optical networks. In this work, we focus on applications of such devices and discuss recent results.
\end{abstract}

\section{INTRODUCTION}

The use of multiple wavelength channels in an optical network introduces an extra degree of freedom. However, this happens at the cost of increased network complexity, in terms of the need to perform wavelength multiplexing, wavelength-routing etc. In addition, the ever increasing demand regarding system capacity and the need to manage the network cost-effectively, for example by allowing service providers to deploy bandwidth when and where it is needed, push for more intelligent network elements. One technology that will enable a step to meet this goal is tunable optical components, more specifically the ability to modify a functionality of an optical component by a control signal, for example heat, voltage, or another light beam.

The concept of microstructured optical fibers filled with liquids was first demonstrated by Eggleton et al. $[1,2]$, where the thermal properties of polymers and high-index oils were utilized to demonstrate tunable waveguiding properties of the fibers. In this work, we focus on components based on microstructured optical fibers infiltrated with liquid crystals - so-called liquid crystal photonic bandgap fibers (LCPBGs) [3]. By utilizing this approach, functionalities such as switching, spectral filtering, attenuation, polarization filtering and polarization control etc. become tunable through the use of liquid crystals. These functions can form the basic building-blocks of more complex devices. The property that allows tunability and dynamic behaviour is the highly sensitive nature of the refractive index of liquid crystals. Changing the temperature of the liquid crystal or/and applying electrical or optical fields can alter these. In the following, we demonstrate how the transmission properties of a microstructured optical fiber may be altered through the use of liquid crystals.

\section{LIQUID CRYSTAL PHOTONIC BANDGAP FIBERS}

Figure 1 shows an optical micrograph of a typical microstructured optical fiber having a central core region surrounded by 7 rings of air holes arranged in a triangular lattice. The core size, inter hole distance, hole diameter and outer diameter are $10 \mu \mathrm{m}, 7 \mu \mathrm{m}, 3 \mu \mathrm{m}$ and $125 \mu \mathrm{m}$, respectively. This type of fiber is a so-called index-guiding fiber, which means that the core has a higher refractive index than the cladding and, therefore, guides light by the principle of Total-Internal-Reflection (TIR), in analogy with standard optical fibers.

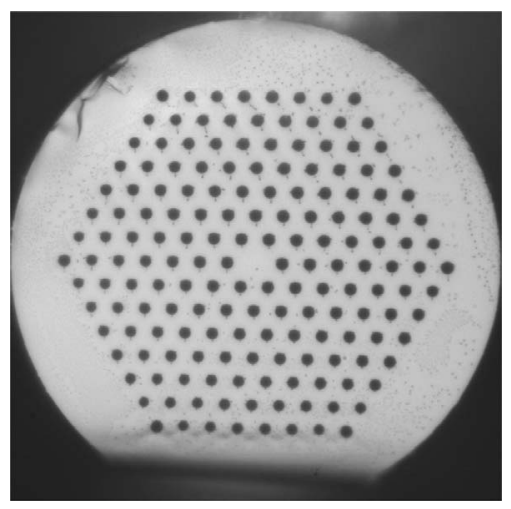

Figure 1. Optical micrograph of the end facet of a PCF. The central core region is surrounded by 7 rings of air holes arranged in a triangular lattice. The inter hole distance and hole diameter are $7 \mu \mathrm{m}$ and $3 \mu \mathrm{m}$, respectively. Picture kindly provided by Crystal Fibre A/S.

Infiltrating the air holes of the silica microstructured optical fiber with a suitable LC having a higher refractive index than that of silica, eliminates the possibility of TIR guidance, since the core is now a low index region. Instead, light can be confined to the core through the photonic bandgap (PBG) effect, where light is reflected 
from the LC filled holes through coherent scattering. A key feature of PBG guidance is the strong wavelength dependent transmission giving rise to transmission bands separated by stopbands.

The novel feature of this type of fiber structure is that the waveguiding properties now are very dependent on the properties of the LC, which can be perturbed relatively easy, resulting in tuning of the fiber properties [3].

\subsection{Spectral filtering and switching}

LC's exhibit a minimum of two phases, where the LC molecules align very differently, some types of LC's exhibit three or more phases. The phases are temperature sensitive meaning that it is possible to switch between phases by varying the temperature. Figure 2 shows the temperature dependent transmission spectrum of the fiber of Figure 1 filled with such a LC. In one phase, the spectrum consists of three transmission bands (the bandgaps) where light is transmitted through the fiber with a loss as low as $1 \mathrm{~dB}$.

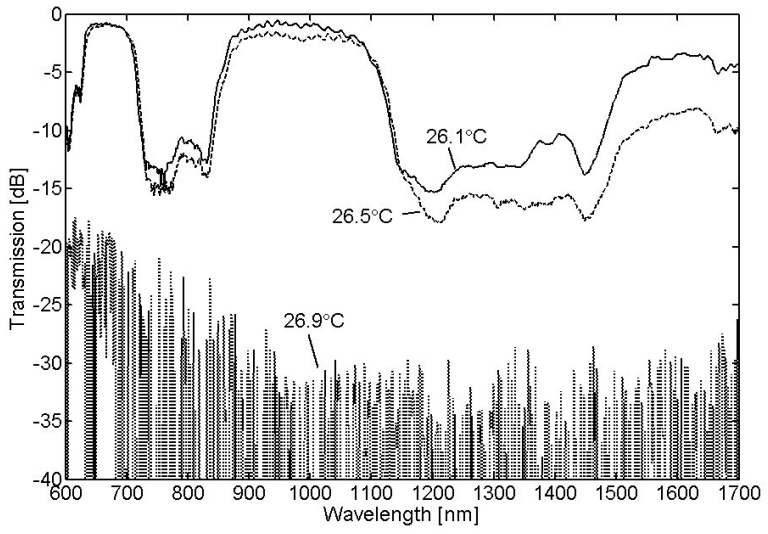

Figure 2. Transmission spectrum of the microstructured optical fiber shown on figure 1 infiltrated for $10 \mathrm{~mm}$ of its length with the LC TM216 (BDH, United Kingdom). The spectrum is shown for three closely-spaced temperatures near a phase transition.

When the temperature is increased by $0.4{ }^{\circ} \mathrm{C}$, in Figure 2 from 26.5 to $26.9^{\circ} \mathrm{C}$, the $\mathrm{LC}$ switches into another phase and very high loss is introduced in the transmission bands forming a switching functionality. Highly sensitive measurements at $\lambda=978 \mathrm{~nm}$ show an extinction ratio up to $80 \mathrm{~dB}$ [4] The device principle is illustrated in Figure 3, showing a schematic of a liquid crystal infiltrated microstructured optical fiber coated with a thin conducting layer acting as a resistive microheater, which allows switching between the two states using only $25 \mu \mathrm{W}$ of electrical power. In this case, the use of LC phase switching results in a step-like response to temperature, and the loss is increased from $1 \mathrm{~dB}$ to $80 \mathrm{~dB}$ within a temperature change of a few tens of a degree Celsius.

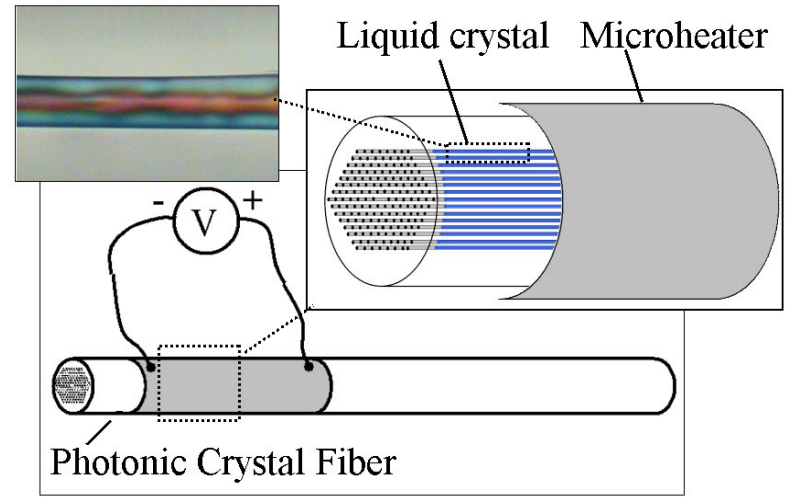

Figure 3. Device principle of a PCF infiltrated with LC. Thermal control is achieved by coating the filled section with a thin conducting layer, which acts as a resistive microheater.

Using other LC's, more continuously tunable functionalities can be obtained, as shown in Figure 4. This figure shows the tunability of a transmission band around $\lambda=545 \mathrm{~nm}$, where the temperature is varied. In this case only $0.5 \mathrm{~dB}$ loss is observed and the spectral position of the transmission band can be tuned $18 \mathrm{~nm}$ over a $12^{\circ} \mathrm{C}$ temperature interval. A much wider transmission band $(\Delta \lambda \approx 400 \mathrm{~nm})$ is also present around $\lambda=1400 \mathrm{~nm}$ (not shown) and the spectral position of this band can be tuned $46 \mathrm{~nm}$ over the same temperature range.

The response time of thermally controlled devices is, of course, limited by the speed at which the temperature can be controlled accurately and the temperature interval at which it should be operated. The response time of the spectral filtering devices is around $1-2 \mathrm{~s}$, while the switching device could be operated somewhat faster (hundreds of $\mathrm{ms}$ ). The thermal response time can be decreased considerably by dye-doping the LC and using pump light to heat the LC. A response time of $100 \mu$ s has been demonstrated using a $\lambda=532 \mathrm{~nm}$ pump beam with a peak power of $2-3 \mathrm{~mW}$ to heat dye-doped E7 [5]. 


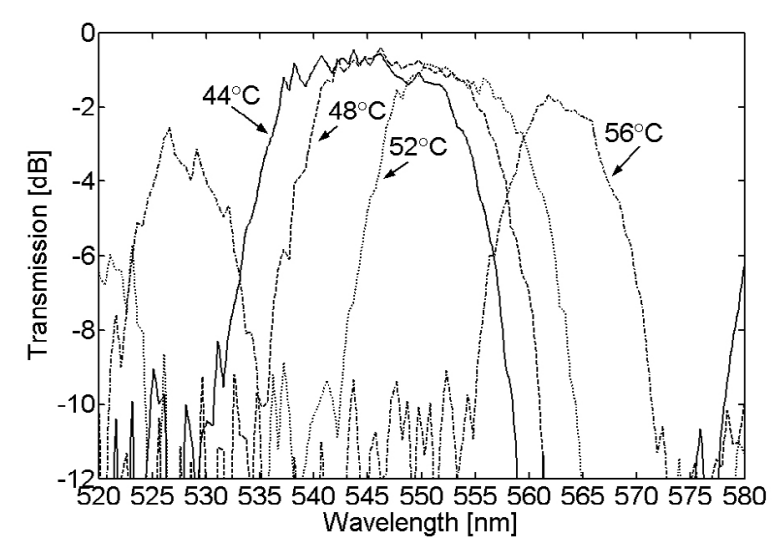

Figure 4. Temperature dependent transmission spectrum of the PCF shown on figure 1 infiltrated for $10 \mathrm{~mm}$ of its length with the LC E7 (Merck, Darmstadt, Germany).

\subsection{Controlling polarization}

LC's exhibit very large electro-optic effect and significant changes in refractive index can be induced by applying an electrical field across the LC. The induced change is caused by a dielectric torque acting on the LC molecules, which reorient accordingly to the field. The index change is anisotropic meaning that the refractive index change is polarization dependent. In LCPBG fibers this gives rise to unique properties where the transmission bands can be electrically shifted [6] as shown in Figure 5.

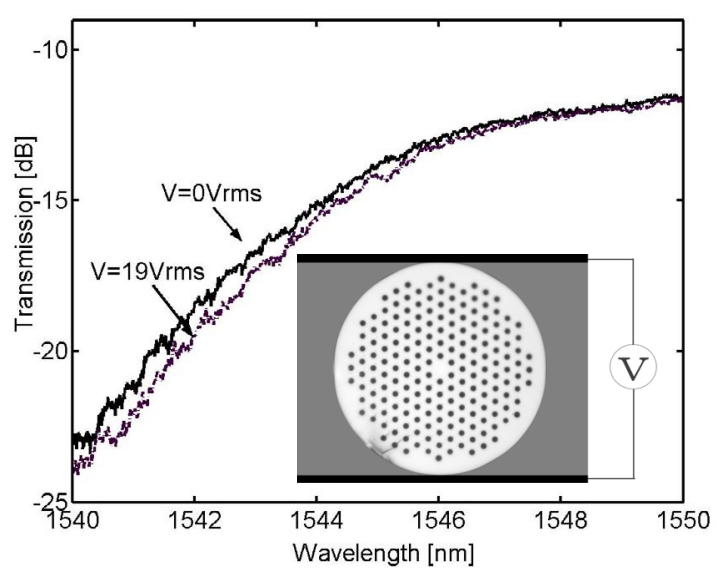

Figure 5. Electro-optic tuning of the bandgap edge of a PCF infiltrated with the dual-frequency nematic LC MDA-00-3639 (Merck, Darmstadt, Germany). The bandgap edge is shown for $V=0 \mathrm{~V}$ and $V=19$ Vrms. A $0.3 \mathrm{~nm}$ shift is observed. Inset illustrates the cross-section of the $\angle C P B G$ fiber sandwiched between two electrodes.

A LCPBG fiber is sandwiched between two electrodes (Figure 5, inset) and a voltage having a frequency of $1 \mathrm{kHz}$ is applied. At $\mathrm{V}=19 \mathrm{Vrms}$ a small bandgap shift of $0.3 \mathrm{~nm}$ is observed, which is polarization dependent meaning that the shift is not as clearly observed for the orthogonal polarization. The polarization splitting of the bandgaps causes the guided modes to loose their degeneracy and, therefore, experience birefringence, which increases as the bandgaps is shifted more. The induced birefringence as a function of applied voltage is shown on Figure 6. The inset shows the output polarization plotted on the Poincaré sphere before and after an applied voltage of $35 \mathrm{Vrms}$. As observed, applying an electrical field induces birefringence, which can rotate the state of polarization.

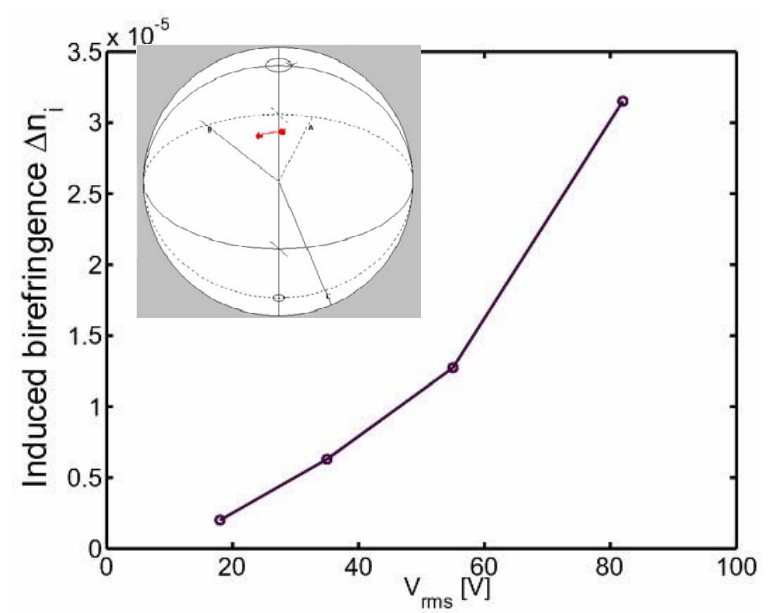

Figure 6. Induced birefringence as a function of applied voltage at $\lambda=1600 \mathrm{~nm}$. Inset shows the output polarization of the $L C P B G$ fiber plotted on the Poincare sphere with an applied voltage of $V=0 \mathrm{~V}$ and $V=35$ Vrms. 
Electrical controlled polarization filtering using LCPBGs has also been demonstrated [7], where the fiber acted as a switchable polarizing filter, with controllable polarization dependent loss. Electrically controlled devices have response times in the $1-10 \mathrm{~ms}$ range for operating voltages less than $100 \mathrm{~V}$, where higher voltage gives the fastest response time.

\subsection{Network components}

Owing to the limited switching speed of the current generation of tunable liquid crystal devices, applications will primarily focus on adaptive power equalization as well as polarization control and optical signal-to-noise ratio monitoring.

Compact tunable optical variable attenuators can be used for channel power equalization as they allow us to compensate for erbium-doped fibre amplifier gain-tilts induced by reconfiguration in a network. Alternatively, they can be applied to channel pre-emphasis at the transmitter. Birefringent elements can be incorporated as quarter and half-wave plates into tunable polarization controllers. Such devices could find use in response to slow polarization drifts prior to polarization dependent devices such as semiconductor optical amplifiers [8]. Furthermore, the implementation of a polarization extinction technique allows for easy and cost effective optical signal-to-noise ratio monitoring in order to e.g. detect amplifier failure over a link [9]. Such a method has been shown to be effective even in the presence of polarization mode dispersion and in-band crosstalk [10]. A tunable polarization controller is also an essential part of polarization mode dispersion compensators and emulators [11]. For all those applications, simultaneous control of the birefringence and attenuation needs to be ensured. Finally, the realisation of tunable photonic crystal fiber based grating filters could be envisaged, for example in the context of adaptive dispersion compensation.

\section{CONCLUSION}

Microstructured optical fiber technology combined with liquid crystals is a promising candidate for creating a platform for tunable fiber devices, which forms various functionalities ranging from polarization control and filtering, spectral filtering and shaping and possibly dispersion trimming. These devices have response time in the millisecond regime and can be tuned by a variety of parameters. The devices have the potential of being small and compact and exhibit low insertion loss.

\section{REFERENCES}

[1] B.J. Eggleton, C. Kerbage, P.S. Westbrook, R. Windeler \& A. Hale, "Microstructured optical fiber devices”, Opt. Express 9, pp. 698-713 (2001).

[2] R.T. Bise, R.S. Windeler, K.S. Kranz, C. Kerbage, B.J. Eggleton, and D.J. Trevor, "Tunable photonic band gap fiber," in OSA Trends in Optics and Photonics (TOPS) vol. 70, Optical Fiber Communication Conference Technical Digest, Postconference Edition (Optical Society of America, Washington, DC, 2002), pp. 466-468.

[3] T.T. Larsen, A. Bjarklev, D.S. Hermann, and J. Broeng, "Optical devices based on liquid crystal photonic bandgap fibres," Opt. Express 11, pp. 2589-2596 (2003).

[4] T.T. Larsen, A. Bjarklev, D.S. Hermann, and J. Broeng, "Thermo-optics switching in liquid crystal infiltrated photonic crystal fibers", Electronics letters 39, pp. 1719-1720 (2003).

[5] T.T. Alkeskjold, J. Lægsgaard, A. Bjarklev, D.S. Hermann, Anawati, J. Broeng, Jun Li And Shin-Tson $\mathrm{Wu}$, “All-optical modulation in dye-doped nematic liquid crystal photonic bandgap fibers," Opt. Express 12, pp. 5857-5871 (2004), http://www.opticsexpress.org/abstract.cfm?URI=OPEX-12-24-5857.

[6] L. Scolari, T.T. Alkeskjold, J. Riishede, M.D. Nielsen, A. Bjarklev, D.S. Hermann, A. Anawati, P. Bassi, "Electrically Controllable Liquid Crystal Photonic Bandgap Fiber with Dual-Frequency Control", To be presented at the Workshop of Fibers and Optical Passive Components(WFOPC), Palermo, Italy, June 2005.

[7] M.W. Haakestad, T.T. Alkeskjold, M.D. Nielsen, L. Scolari, J. Riishede, H.E. Engan and A. Bjarklev, "Electrically Tunable Photonic Bandgap Guidance in a Liquid Crystal Filled Photonic Crystal Fiber,' IEEE Phot. Tech. Lett. 17, pp. 819-821 (2005).

[8] T. Chiba, Y. Ohtera and S. Kawakami, "Polarization stabilizer using liquid crystal rotatable waveplates", J. Lightwave Technol. 17, pp. 885-890, 1999.

[9] J. H. Lee, D.K. Jung, C.H.Kim and Y.C. Chung, "OSNR monitoring technique using polarization-nulling method”, IEEE Photon. Technol. Lett. 13, pp. 88-90, 2001.

[10] M.-H. Cheung, L.-K. Chen and C.-K. Chan, "PMD-insensitive OSNR monitoring based on polarizationnulling with off-center narrow-band filtering”, IEEE Photon. Technol. Lett. 16, pp. 2562-2564, 2004.

[11] A.E. Willner, S.M. Reza Motaghian Nezam, L. Yan, Z. Pan and M.C. Hauer, "Monitoring and control of polarization-related impairments in optical fiber systems”, J. Lightwave Technol. 22, pp. 106-125, 2004. 